NUMER, FUNCT, ANAL. AND OPTIMIZ., 9(3\&4), 381-414 (1987)

\title{
CENTER MANIFOLDS OF DYNAMICAL SYSTEMS UNDER DISCRETIZATION
}

\author{
by \\ Wolf-Jürgen Beyn \\ Fakultät für Mathematik \\ Universitāt Konstanz, W. Germany \\ and \\ Jens Lorenz \\ Applied Mathematics 217-50 \\ California Institute of Technology \\ Pasadena, CA 91125 U.S.A.
}

\begin{abstract}
We consider an autonomous dynamical system discretized by a one-step method. The point $z=0$ is assumed to be fixed under the continuous and the discrete flows. We allow $z=0$ to be non-hyperbolic. The continuous system has a center-unstable manifold and we show the existence of approximating invariant manifolds for the discretizations. The manifolds for the continuous and the discrete systems share the property of being locally attracting at an exponential rate; the dynamics inside the manifolds can differ qualitatively, however, for all step-sizes $h$.
\end{abstract}

1. INTRODUCTION. Consider an autonomous system

$$
d z / d t=f(z), \quad z(t) \in \mathbf{R}^{n}
$$

discretized by a one-step method

$$
z_{j+1}=\phi\left(z_{j}, h, f\right), \quad j=0,1 \ldots
$$

with uniform step-size $h$. Under very general conditions individual trajectories of the continuous-time system (1.1) are well approximated by (1.2) if 
the time interval of consideration is not too long. However, over long time intervals the error will, in general, grow exponentially with time and any accuracy of the approximation will finally be lost. Thus it is a fundamental question if - and in what sense - the discrete dynamical systems (1.2) approximate (1.1) in cases where long time phenomena in (1.1) are of interest. This paper gives a contribution to this question for the case where (1.1) has a stationary point at $z=0$ which is not necessarily hyperbolic, i.e. the matrix $f^{\prime}(0)$ may have eigenvalues with zero real-part. If $z=0$ is hyperbolic, then the dynamics of (1.1) near 0 is well understood. For this case Beyn [1] has shown in what sense (1.2) approximates (1.1) as $h \rightarrow 0$ under quite general conditions. The case of a non-hyperbolic fixed point is much more complicated, however, and indeed our analysis given here is much less complete; it can only be considered as a starting point.

A fundamental tool used in the theory of dynamical systems is the center manifold theorem which states - in non-technical terms - that one can 'split off' the influence of the stable and the unstable eigenvalues; more precisely, (1.1) has a local invariant manifold, a so-called center manifold, tangent to the sum of the generalized eigenspaces to the eigenvalues with real-part equal zero. In the directions of stable or unstable eigenspaces the flow of (1.1) is exponentially attracting or repelling, respectively. Inside the center manifold the dynamic can be very complicated, however.

The exponential behavior of the flow of (1.1) near the center manifold makes it plausible that the manifold persists under small perturbations, and thus one can also expect the existence of approximating manifolds in (1.2) for small $h$. It is the aim of this paper to establish such a result. (For perturbation results of invariant manifolds in the theory of ordinary differential equations, see Fenichel [3]). We will say nothing, however, about the comparative dynamics inside the center manifold and its approximations.

The formulation of our result is complicated by the fact that the center manifold for (1.1) is usually not unique, though all center manifolds are tangent to each other at $z=0$ to all orders; see Sijbrand [5] for an enlightening discussion. To keep the technical difficulties of this paper limited we will actually establish our result for the center-unstable manifolds instead of the center manifolds. Of course, if $f^{\prime}(0)$ has no unstable eigenvalues, there 
is no difference. If there are unstable eigenvalues, however, then a treatment of the center manifolds would require an additional split-off of the unstable subspace. This could be done using the same techniques as described below.

We now give a more technical outline of the paper. The smooth function $f(z)$ is assumed to have the form

$$
f(z)=L z+N(z), \quad\|N(z)\|=\mathrm{O}\left(\|z\|^{2}\right)
$$

with a linear operator $L$ which has complementary invariant subspaces $X$ and $Y$ :

$$
\begin{aligned}
& L(X) \subset X, \quad L(Y) \subset Y, \quad \mathbb{R}^{n}=X \oplus Y, \\
& A:=\left.L\right|_{X}, \quad \operatorname{Re} \sigma(A) \geq 0, \\
& B:=\left.L\right|_{Y}, \quad \operatorname{Re} \sigma(B)<0 .
\end{aligned}
$$

Thus all eigenvalues of $A$ have nonnegative real-parts. It is known that one can construct local center-unstable manifolds for (1.1) of the form

$$
M_{\epsilon, \mathrm{loc}}=\left\{\left(x, u_{\epsilon}(x)\right): x \in X,\|x\|<\epsilon\right\} .
$$

Each manifold $M_{\epsilon, \text { loc }}$ is locally invariant under the flow of the differential equaiton (1.1) and the function $u_{\epsilon}: X \rightarrow Y$ satisfies $u_{\epsilon}(0)=0, u_{\epsilon}^{\prime}(0)=0$. (Indeed, the existence of such manifolds will follow from our results.)

After discussion of the (weak) assumption for the one-step method in section 2 we describe certain cut-off and scaling processes which we apply to (1.1) and (1.2). Then the main results are formulated, namely the existence of the center-unstable manifolds $M_{\epsilon, \text { loc }}$ for (1.1) and of approximating invariant manifolds $M_{\epsilon, h, \text { loc }}$ for (1.2). We like to emphasize here that we do not assume

$$
\operatorname{Re} \lambda=0 \text { implies }|\gamma(h \lambda)|=1
$$

for the growth-function $\gamma$ of the one-step method. Thus eigenvalues $\lambda$ of $L=f^{\prime}(0)$ with $\operatorname{Re} \lambda=0$ might lead to stable or unstable eigenvalues for the discrete dynamical systems (1.2), and consequently $M_{\epsilon, h, \text { loc }}$ is not necessarily a center-unstable manifold for (1.2). If one wants to compare the long time dynamics inside $M_{\epsilon, \text { loc }}$ and $M_{\epsilon, h, \text { loc }}$, however, it seems advisable 
to insist on the implication (1.6). (The term 'growth-function' of a method will be explained in section 7 .)

The existence proof for invariant manifolds is given in section 4 using the graph transform technique. We investigate the influence of the discretization parameter $h$. The existence proof applies to (1.2) and also to the $h$-flow of the differential equation (1.1). Due to the semigroup property of the differential-equation-flow the latter manifolds turn out to be independent of $h$. This establishes the existence of the center-unstable manifolds $M_{\epsilon, \text { loc }}$ for (1.1). Though this result is known we indicate its proof in section 5 for completeness. Section 6 proves the error estimates as $h \rightarrow 0$. In section 7 we give additional information about the invariant manifolds for the discrete systems. First it is shown that they are exponentially attracting. This allows us to sharpen - under special assumptions - a recent result of Kloeden and Lorenz [4] about uniformly attracting sets $\boldsymbol{A}$ for differential equations and corresponding sets $\Lambda(h)$ of their one-step discretizations.

\section{ASSUMPTIONS FOR THE ONE-STEP METHOD}

To illustrate the - rather weak - assumptions we use $m$-stage explicit Runge-Kutta methods; for these the function $\phi(z, h, f)$ of (1.2) has the form

$$
\phi(z, h, f)=z+h \sum_{i=0}^{m} \beta_{i} k_{i}(z, h, f)
$$

with $k_{0}(z, h, f)=f(z)$ and

$$
k_{i}(z, h, f)=f\left(z+h \sum_{j=0}^{i-1} \beta_{i j} k_{j}(z, h, f)\right), \quad i=1, \ldots, m
$$

The numbers $\beta_{i}, \beta_{i j}$ are constants defining the specific method. E.g., the standard fourth order Runge-Kutta method is obtained for

$$
\begin{aligned}
& m=3, \quad \beta_{0}=\beta_{3}=\frac{1}{6}, \quad \beta_{1}=\beta_{2}=\frac{1}{3} \\
& \beta_{10}=\beta_{21}=\frac{1}{2}, \quad \beta_{32}=1, \quad \beta_{20}=\beta_{30}=\beta_{31}=0
\end{aligned}
$$


We will assume that the function $f: \mathbb{R}^{n} \rightarrow \mathbb{R}^{n}$ of (1.1) is globally defined, for simplicity; also let $f \in C^{p+k+2}$. The number $p \geq 1$ will denote the order of the one-step method whereas $k \geq 0$ denotes the number of derivatives for which convergence of the manifolds will be obtained. We did not try to minimize smoothness assumptions for $f$. In the following $c_{1}, c_{2}$ etc. will denote positive constants independent of the step-size $h, 0 \leq h \leq h_{0}$. The step-size $h_{0}$ will not necessarily be the same at different appearances.

To ensure that $z=0$ stays fixed for (1.2) assume

$$
\phi(0, h, f)=0 \text { for } 0 \leq h \leq h_{0} \text { if } f(0)=0 \text {. }
$$

Assumption (A0) and also the following two technical assumptions are easily seen to be fulfilled for all methods (2.1).

(A1) Given $c_{1}$ there exists $c_{2}$ and $h_{0}>0$ such that

$$
\frac{\partial^{p+1+|\alpha|}}{\partial h^{p+1} \partial z^{\alpha}} \phi(z, h, f)
$$

exists for $|\alpha| \leq k+1,\|z\| \leq c_{1}, \quad 0 \leq h \leq h_{0}$, is continuous at these arguments and can be estimated by

$$
c_{2}\left\{1+\sup \left\{\left\|\frac{\partial^{|\alpha|}}{\partial z^{\alpha}} f(z)\right\|,|\alpha| \leq p+k+2,\|z\| \leq 2 c_{1}\right\}\right\} .
$$

(A2) The value $\phi(z, h, f)$ only depends on $f$-values in an $O(h)$ neighborhood of $z$. More precisely, given $c_{1}$ there exist $c_{2}$ and $h_{0}$ such that

$$
\phi(z, h, f)=\phi(z, h, g), \quad 0 \leq h \leq h_{0},
$$

whenever $\|z\| \leq c_{1}$ and

$$
f(s)=g(\varsigma) \text { for }\|z-\varsigma\| \leq c_{2} h
$$

With $\Phi(z, t, f)$, we denote the flow of (1.1); i.e. the solution of (1.1) subject to the initial condition $z(0)=z_{0}$ is

$$
z(t)=\Phi\left(z_{0}, t, f\right),|t|<t_{0}\left(z_{0}\right)
$$


The usual formulation of $p$-th order accuracy of the method (1.2) is

$$
\|\phi(z, h, f)-\Phi(z, h, f)\|=0\left(h^{p+1}\right)
$$

Under our smoothness assumptions this is equivalent to

$$
\begin{aligned}
& \frac{\partial^{i}}{\partial h^{i}} \phi(z, 0, f)=\frac{\partial^{i}}{\partial h^{i}} \Phi(z, 0, f) \\
& \text { for } i=0, \ldots, p \text { and all } z \in \mathbb{R}^{n} \text {. }
\end{aligned}
$$

As is well known, fulfillment of (A3) for the methods (2.1) depends on the specific choice of the constants $\beta_{i}, \beta_{i j}$. For example, the standard Runge-Kutta method fulfills (A3) with $p=4$.

A final assumption, which will simplify an argument below, is invariance of the one-step method with respect to scalings of $f$ :

(A4) If $\epsilon \neq 0$ and $\tilde{f}(\tilde{z})=\frac{1}{\epsilon} f(\epsilon \tilde{z}), \tilde{z} \in \mathbb{R}^{n}$, then $\phi(\tilde{z}, h, \tilde{f})=\frac{1}{\epsilon} \phi(\epsilon \tilde{z}, h, f)$. To see that (A4) is fulfilled for all methods (2.1) first note

$$
k_{0}(\tilde{z}, h, \tilde{f})=\tilde{f}(\tilde{z})=\frac{1}{\epsilon} f(\epsilon \tilde{z})=\frac{1}{\epsilon} k_{0}(\epsilon \tilde{z}, h, f) .
$$

Then proceed inductively:

$$
\begin{aligned}
k_{i}(\tilde{z}, h, \tilde{f}) & =\tilde{f}\left(\tilde{z}+h \sum_{j=0}^{i-1} \beta_{i j} k_{j}(\tilde{z}, h, \tilde{f})\right) \\
& =\tilde{f}\left(\tilde{z}+h \frac{1}{\epsilon} \sum_{j=0}^{i-1} \beta_{i j} k_{j}(\epsilon \tilde{z}, h, f)\right) \\
& =\frac{1}{\epsilon} f\left(\epsilon \tilde{z}+h \sum_{j=0}^{i-1} \beta_{i j} k_{j}(\epsilon \tilde{z}, h, f)\right) \\
& =\frac{1}{\epsilon} k_{i}(\epsilon \tilde{z}, h, f) .
\end{aligned}
$$

Thus (A4) follows from (2.1).

For other classes of methods, like implicit Runge-Kutta methods, for example, the assumptions (AO) to (A4) are also easily established. 


\section{ESTIMATES FOR FLOWS AND FORMULATION OF THE MAIN}

\section{RESULTS.}

In this section we describe certain cut-off and scaling processes applied to (1.1), (1.2) and show two technical lemmata about the modified flows. A global existence and convergence result for invariant manifolds will be proved in sections 4 and 6 for the modified flows; reinterpretation in terms of the original systems (1.1), (1.2) leads to local results which we formulate in Theorems 3.8, 3.9 and 3.10. We always assume (1.3), (1.4) for the differential equation and conditions (A0) to (A4) for the one-step method. The following lemma is a consequence of (1.4):

Lemma 3.1 1. There exists $0<\beta \leq 1$ and a norm \|\|$_{Y}$ on $Y$ such that

$$
\left\|e^{B h}\right\|_{Y} \leq 1-\beta h \text { for } 0 \leq h \leq h_{0} .
$$

2. Given $N_{1}>0$ there exists a norm \|\|$_{X}$ on $X$ such that

$$
\left\|e^{-A h}\right\|_{X} \leq 1+\frac{\beta}{N_{1}} h \text { for } 0 \leq h \leq h_{0} .
$$

The number $N_{1}$ and hence \|\|$_{X}$ will be chosen appropriately in the existence proof of theorem 4.1. The norm for $z=(x, y) \in \mathbb{R}^{n}$ is always defined by

$$
\|z\|=\max \left\{\|x\|_{X},\|y\|_{Y}\right\}
$$

and henceforth we drop the indices $X$ and $Y$.

Let $\psi: \mathbb{R}^{n} \rightarrow \mathbb{R}$ denote a $C^{\infty}$ cut-off function, i.e.

$$
0 \leq \psi(z) \leq 1, \quad \psi(z)=\left\{\begin{array}{ll}
1 & \text { for }\|z\| \leq 1 \\
0 & \text { for }\|z\| \geq 2
\end{array}, z \in \mathbb{R}^{n} .\right.
$$

We define cut-off systems to (1.1) by

$$
d z / d t=L z+\psi(z / \epsilon) N(z)=: f_{\epsilon}(z), \quad 0<\epsilon \leq 1 .
$$

The flows $\Phi\left(z, t, f_{\epsilon}\right)$ are defined for all $t$. It is convenient to introduce the scaled variables $\tilde{z}=z / \epsilon$ for which the cut-off system (3.1) reads

$$
d \tilde{z} / d t=\tilde{f}_{\epsilon}(\tilde{z}), \quad \tilde{f}_{\epsilon}(\tilde{z})=\frac{1}{\epsilon} f_{\epsilon}(\epsilon \tilde{z})
$$


One easily checks that the flows scale similarly:

$$
\Phi\left(\tilde{z}, t, \tilde{f}_{\epsilon}\right)=\frac{1}{\epsilon} \Phi\left(\epsilon \tilde{z}, t, f_{\epsilon}\right)
$$

The flows for (3.2) have the following property:

Lemma 3.2 There are positive constants $C$ and $K$ independent of $0 \leq$ $t \leq 1$ and $0<\epsilon \leq 1$ such that

$$
\Phi\left(\tilde{z}, t, \tilde{f}_{\epsilon}\right)=e^{L t} \tilde{z}+\rho(\tilde{z}, t, \epsilon), \tilde{z} \in \mathbb{R}^{n}
$$

with

$$
\rho(\tilde{z}, t, \epsilon)=0 \text { for }\|\tilde{z}\| \geq K
$$

$$
\left\|\frac{\partial^{|\alpha|}}{\partial \tilde{z}^{\alpha}} \rho(\tilde{z}, t, \epsilon)\right\| \leq C \epsilon t \text { for } \tilde{z} \in \mathbb{R}^{n},|\alpha| \leq k+1 .
$$

Proof: 1. First note

$$
\tilde{f}_{\epsilon}(\tilde{z})=L \tilde{z}+\frac{1}{\epsilon} \psi(\tilde{z}) N(\epsilon \tilde{z})
$$

thus $\tilde{f}_{\epsilon}(\tilde{z})=L \tilde{z}$ for $\|\tilde{z}\| \geq 2$.

Define

$$
c:=\min _{0 \leq t \leq 1} \frac{1}{\left\|e^{-L t}\right\|}>0
$$

and set $K=2 / c$. If $\|\tilde{z}\| \geq K$ and $0 \leq t \leq 1$, then

$$
K \leq\|\tilde{z}\| \leq\left\|e^{-L t}\right\| \quad\left\|e^{L t} \tilde{z}\right\|
$$

Thus $\left\|e^{L t} \tilde{z}\right\| \geq K c=2$, and therefore

$$
\Phi\left(\tilde{z}, t, \tilde{f}_{\epsilon}\right)=e^{L t} \tilde{z}, \quad\|\tilde{z}\| \geq K, \quad 0 \leq t \leq 1
$$

This demonstrates (3.4).

2. With $r_{\epsilon}(\tilde{z}):=\frac{1}{\epsilon} \psi(\tilde{z}) N(\epsilon \tilde{z})$ we can write

$$
\tilde{f}_{\epsilon}(\tilde{z})=L \tilde{z}+r_{\epsilon}(\tilde{z})
$$


by (3.6). Note that $r_{\epsilon}(\tilde{z}) \equiv 0$ for $\|\tilde{z}\| \geq 2$. Hence the assumption $\|N(z)\|=0\left(\|z\|^{2}\right)$ implies

$$
\left\|r_{\epsilon}(\tilde{z})\right\| \leq \frac{1}{\epsilon} c_{1} \epsilon^{2}=c_{1} \epsilon, \tilde{z} \in \mathbb{R}^{n}
$$

To estimate derivatives of $r_{\epsilon}$ use Leibniz' rule and find

$$
\left\|\frac{\partial^{|\alpha|}}{\partial \tilde{z}^{\alpha}} r_{\epsilon}(\tilde{z})\right\| \leq c_{1} \epsilon, \quad \tilde{z} \in \mathbb{R}^{n},|\alpha| \leq k+1
$$

3. We now show (3.5) first for $\alpha=0$ and thus compare

$$
\Phi_{1}(t)=\Phi\left(\tilde{z}, t, \tilde{f}_{\epsilon}\right) \text { with } \Phi_{2}(t)=\Phi(\tilde{z}, t, L)=e^{L t} \tilde{z}
$$

The difference

$$
\delta(t)=\Phi_{2}(t)-\Phi_{1}(t)
$$

satisfies $\delta(0)=0$ and

$$
\begin{aligned}
\delta^{\prime}(t) & =L \Phi_{1}(t)+r_{\epsilon}\left(\Phi_{1}(t)\right)-L \Phi_{2}(t) \\
& =L \delta(t)+r_{\epsilon}\left(\Phi_{1}(t)\right) .
\end{aligned}
$$

Hence

$$
\begin{aligned}
\|\delta(t)\| & =\left\|\int_{0}^{t} e^{L(t-s)} r_{\epsilon}\left(\Phi_{1}(s)\right) d s\right\| \\
& \leq c_{2} \epsilon \int_{0}^{t} e^{\gamma(t-s)} d s \leq c_{3} \epsilon t .
\end{aligned}
$$

This shows (3.5) for $\alpha=0$.

4. To obtain estimates for derivatives of $\rho(\tilde{z}, t, \epsilon)=\Phi\left(\tilde{z}, t, f_{\epsilon}\right)-e^{L t} \tilde{z}$ w.r.t. $\tilde{z}$ note that the function

$$
\sigma(t):=\frac{\partial^{|\alpha|}}{\partial \tilde{z}^{\alpha}} \rho(\tilde{z}, t, \epsilon)
$$

fulfills $\sigma(0)=0$ and

$$
\sigma^{\prime}(t)=L \sigma(t)+\frac{\partial^{|\alpha|}}{\partial \tilde{z}^{\alpha}}\left\{r_{\epsilon}\left(\Phi\left(\tilde{z}, t, \tilde{f}_{\epsilon}\right)\right)\right\} .
$$


Since $\tilde{f}_{\epsilon}$ differs from $r_{\epsilon}$ only by a linear function, it follows from (3.7) that the derivatives of $\tilde{f}_{\epsilon}$ are uniformly bounded. As is well known, this implies uniform boundedness of the corresponding derivatives of the flow $\Phi\left(\tilde{z}, t, \tilde{f}_{\epsilon}\right)$ w.r.t. $\tilde{z}$. But then (3.7) shows that the term

$$
\frac{\partial^{|\alpha|}}{\partial \tilde{z}^{\alpha}}\{\ldots\}
$$

in the equation for $\sigma(t)$ is $O(\epsilon)$. Thus an estimate $\|\sigma(t)\| \leq c t$ follows in the same way as the estimate for $\delta(t)$.

We need similar estimates for the discrete-time flow $\Phi\left(\tilde{z}, h, \tilde{f}_{\epsilon}\right)$ of the one-step method applied to the scaled cut-off systems (3.2).

Lemma 3.3 Let $K$ denote a given constant. There exists another constant $C=C(K)$ independent of $0 \leq h \leq h_{0}$ and $0<\epsilon \leq 1$ such that

$$
\phi\left(\tilde{z}, h, \tilde{f}_{\epsilon}\right)=e^{L h} \tilde{z}+\sigma(\tilde{z}, h, \epsilon), \quad \tilde{z} \in \mathbb{R}^{n},
$$

with

$$
\begin{gathered}
\left\|\frac{\partial^{|\alpha|}}{\partial \tilde{z}^{\alpha}} \sigma(\tilde{z}, h, \epsilon)\right\| \leq C h(\epsilon+h) \\
\text { for }\|\tilde{z}\| \leq 2 K, \quad|\alpha| \leq k+1, \\
\left\|\frac{\partial^{|\alpha|}}{\partial \tilde{z}^{\alpha}} \phi\left(\tilde{z}, h, \tilde{f}_{\epsilon}\right)-\frac{\partial^{|\alpha|}}{\partial \tilde{z}^{\alpha}} \Phi\left(\tilde{z}, h, \tilde{f}_{\epsilon}\right)\right\| \leq C h^{p+1} \\
\text { for }\|\tilde{z}\| \leq 2 K, \quad|\alpha| \leq k+1 .
\end{gathered}
$$

Proof: The estimate (3.9) follows immediately from (3.10) and (3.5) since $p \geq 1$ and

$$
\sigma(\tilde{z}, h, \epsilon)=\rho(\tilde{z}, h, \epsilon)+\phi\left(\tilde{z}, h, \tilde{f}_{\epsilon}\right)-\Phi\left(\tilde{z}, h, \tilde{f}_{\epsilon}\right) .
$$

To show (3.10) we use Taylor expansion at $h=0$ to obtain

$$
\begin{aligned}
\frac{\partial^{|\alpha|}}{\partial \tilde{z}^{\alpha}} \phi\left(\tilde{z}, h, \tilde{f}_{\epsilon}\right) & =\sum_{i=0}^{p} \frac{\partial^{|\alpha|+i}}{\partial \tilde{z}^{\alpha} \partial h^{i}} \phi\left(\tilde{z}, 0, \tilde{f}_{\epsilon}\right) \frac{h^{i}}{i !}+R_{1} \\
& =\sum_{i=0}^{p} \frac{\partial^{|\alpha|+i}}{\partial \tilde{z}^{\alpha} \partial h^{i}} \Phi\left(\tilde{z}, 0, \tilde{f}_{\epsilon}\right) \frac{h^{i}}{i !}+R_{1} \quad(\text { by }(\mathrm{A} 3)) \\
& =\frac{\partial^{|\alpha|}}{\partial \tilde{z}^{\alpha}} \Phi\left(\tilde{z}, h, \tilde{f}_{\epsilon}\right)+R_{1}-R_{2} .
\end{aligned}
$$


$R_{1}-R_{2}$ is the difference of the remainder terms, i.e.

$$
R_{1}-R_{2}=\frac{1}{p !} \int_{0}^{1}(1-s)^{p} \frac{\partial^{|\alpha|+p+1}}{\partial \tilde{z}^{\alpha} \partial h^{p+1}}(\phi-\Phi)\left(\tilde{z}, s h, \tilde{f}_{\epsilon}\right) d s h^{p+1} .
$$

It suffices to show that the integrand stays uniformly bounded. For the $\Phi$ part this follows from the differential equation for $\Phi$ and uniform bounds for derivatives of $\tilde{f}_{\epsilon}$. (Compare part 4 of the proof of lemma 3.2.) For the $\phi$-part boundedness follows from assumption (A1).

It will be convenient to apply an additional cut-off to the discrete flows by setting

$$
\begin{aligned}
w(\tilde{z}, h, \epsilon) & =e^{L h} \tilde{z}+\psi(\tilde{z} / K) \sigma(\tilde{z}, h, \epsilon) \\
& =: e^{L h} \tilde{z}+\mu(\tilde{z}, h, \epsilon)
\end{aligned}
$$

Here $K$ will denote the constant defined in the proof of lemma 3.2. Since $\mu \equiv 0$ for $\|\tilde{z}\| \geq 2 K$ we have by (3.9)

$$
\begin{aligned}
\| \frac{\partial^{|\alpha|}}{\partial \tilde{z}^{\alpha}} & \mu(\tilde{z}, h, \epsilon) \| \leq C^{*} h(\epsilon+h) \\
& \text { for } \tilde{z} \in \mathbb{R}^{n}, \quad|\alpha| \leq k+1, \quad 0 \leq h \leq h_{0}, \quad 0<\epsilon \leq 1 .
\end{aligned}
$$

The $h$-flow of the differential equation (3.2) is

$$
\Phi\left(\tilde{z}, h, \tilde{f}_{\epsilon}\right)=e^{L h} \tilde{z}+\rho(\tilde{z}, h, \epsilon)
$$

$$
\begin{aligned}
& \text { with }\left\|\frac{\partial^{|\alpha|}}{\partial \tilde{z}^{\alpha}} \rho(\tilde{z}, h, \epsilon)\right\| \leq C \epsilon h \\
& \text { for } \tilde{z} \in \mathbb{R}^{n}, \quad|\alpha| \leq k+1, \quad 0 \leq h \leq 1, \quad 0<\epsilon \leq 1 .
\end{aligned}
$$

Also, from (3.4) we find

$$
\psi(\tilde{z} / K) \rho(\tilde{z}, h, \epsilon)=\rho(\tilde{z}, h, \epsilon),
$$

and thus the estimate (3.10) implies a global estimate between $w$ and $\Phi$ :

$$
\begin{aligned}
& \left\|\frac{\partial^{|\alpha|}}{\partial \tilde{z}^{\alpha}} w(\tilde{z}, h, \epsilon)-\frac{\partial^{|\alpha|}}{\partial \tilde{z}^{\alpha}} \Phi\left(\tilde{z}, h, \tilde{f}_{\epsilon}\right)\right\| \leq c_{1} h^{p+1} \\
& \text { for } \tilde{z} \in \mathbb{R}^{n}, \quad|\alpha| \leq k+1, \quad 0 \leq h \leq h_{0}, \quad 0<\epsilon \leq 1 .
\end{aligned}
$$


This summarizes the essential estimates for the modified flows.

We now formulate the main results of the paper. Let

$$
V=\left\{\tilde{u} \in C^{k+1}(X, Y): \tilde{u}(0)=0, \quad\|\tilde{u}\|_{k+1} \leq 1\right\}
$$

where

$$
\|\tilde{u}\|_{k+1}=\sup \left\{\left\|\frac{\partial^{|\alpha|}}{\partial \tilde{x}^{\alpha}} \tilde{u}(\tilde{x})\right\|: \tilde{x} \in X,|\alpha| \leq k+1\right\} .
$$

Let $\bar{V}$ denote the closure of $V$ w.r.t. \|\|$_{k}$, thus

$$
\bar{V}=\left\{\tilde{u} \in C^{k}(X, Y): \tilde{u}(0)=0,\|\tilde{u}\|_{k} \leq 1,\right.
$$

the $k$-th derivatives are Lipschitz bounded with constant 1$\}$.

With a contraction argument w.r.t. \|\|$_{k}$ in $\bar{V}$ we show in section 4:

Lemma 3.4 The flows $w(\tilde{z}, h, \epsilon)$ have a globally defined invariant manifold

$$
\tilde{M}_{\epsilon, h}=\left\{\left(\tilde{x}, \tilde{u}_{\epsilon, h}(\tilde{x})\right): \tilde{x} \in X\right\} \text { for } 0<h \leq h_{0}, \quad 0<\epsilon \leq \epsilon_{0} .
$$

Here $\tilde{u}_{\epsilon, h} \in \bar{V}$.

The proof of the result relies mainly on the estimate (3.12) for the $\mu$ part of the flow

$$
w(\tilde{z}, h, \epsilon)=e^{L h} \tilde{z}+\mu(\tilde{z}, h, \epsilon) .
$$

According to (3.13) the result can also be applied to $\Phi\left(\tilde{z}, h, \tilde{f}_{\epsilon}\right)$. We show in section 5 that the corresponding manifolds do not depend on $h$. This leads to

Lemma 3.5 The flows $\Phi\left(\tilde{z}, h, \tilde{f}_{\epsilon}\right)$ have a globally defined invariant manifold

$$
\tilde{M}_{\epsilon}=\left\{\left(\tilde{x}, \tilde{u}_{\epsilon}(\tilde{x})\right): \tilde{x} \in X\right\} \text { for } 0<\epsilon \leq \epsilon_{0} .
$$

Here $\tilde{u}_{\varepsilon} \in \bar{V}$. In addition, $\tilde{u}_{\epsilon}^{\prime}(0)=0$ if $k \geq 1$; thus $\tilde{M}_{\epsilon}$ is a global centerunstable manifold for the scaled cut-off system (3.2). The global estimate (3.14) allows a comparison of the manifolds $\tilde{M}_{\epsilon}$ and $\tilde{M}_{\epsilon, h}$. In section 6 we prove an error estimate in terms of the defining functions $\tilde{u}_{\epsilon}$ and $\tilde{u}_{\epsilon, h}$ : 
Lemma 3.6 The manifolds constructed satisfy an estimate

$$
\left\|\tilde{u}_{\epsilon}-\tilde{u}_{\epsilon, h}\right\|_{k} \leq C h^{p}
$$

with $C$ independent of $0<h \leq h_{0}, 0<\epsilon \leq \epsilon_{0}$.

The global results formulated in the previous lemmata for the modified flows $w(\tilde{z}, h, \epsilon)$ and $\Phi\left(\tilde{z}, t, \tilde{f}_{\epsilon}\right)$ lead to local results for the given flows $\phi(z, h, \epsilon)$ of the one-step method and $\Phi(z, t, f)$ of the differential equation. The following definition, in which we restrict ourselves to $t \geq 0$, is standard.

Definition 3.7 Let $B_{\epsilon}(0)=\left\{z \in \mathbb{R}^{n}:\|z\|<\epsilon\right\}$ and let $M_{\epsilon, \text { loc }} \subset B_{\epsilon}(0)$ denote a manifold. $M_{\epsilon, \text { loc }}$ is called locally invariant under the flow $\Phi(z, t, f)$ if the following holds: Whenever $z \in M_{\epsilon, \text { loc }}$ and $\Phi(z, t, f) \in B_{\epsilon}(0)$ for $0 \leq t \leq t_{0}$, then $\Phi(z, t, f) \in M_{\epsilon, \text { loc }}$ for $0 \leq t \leq t_{0}$. In other words, trajectories starting on $M_{\epsilon, \text { loc }}$ stay on $M_{\epsilon, \text { loc }}$ as long as they stay in $B_{\epsilon}(0)$.

An analogous definition applies to discrete-time flows $z_{j+1}=\phi\left(z_{j}, h, f\right)$. In the unscaled variables the function $\tilde{u}_{\epsilon}(\tilde{x})$ of lemma 3.5 reads

$$
u_{\epsilon}(x)=\epsilon \tilde{u}_{\epsilon}(x / \epsilon), \quad x \in X,
$$

with $\left\|u_{\epsilon}(x)\right\| \leq \epsilon, u_{\epsilon}(0)=0, u_{\epsilon}^{\prime}(0)=0$. Clearly the manifold

$$
M_{\epsilon}=\left\{\left(x, u_{\epsilon}(x)\right): x \in X\right\}
$$

is globally invariant for the flow $\Phi\left(z, t, f_{\epsilon}\right)$ of the cut-off system (3.1). Since the cut-off process does not effect vectors with norm $\leq \epsilon$ it follows that

$$
\begin{aligned}
& \Phi(z, t, f) \in B_{\epsilon}(0) \text { for } 0 \leq t \leq t_{0} \text { implies } \\
& \Phi(z, t, f)=\Phi\left(z, t, f_{\epsilon}\right) \text { for } 0 \leq t \leq t_{0} .
\end{aligned}
$$

Therefore lemma 3.5 leads to the center-unstable manifold theorem:

\section{Theorem 3.8}

The flow $\Phi(z, t, f)$ of (1.1) has locally invariant manifolds

$$
M_{\epsilon, \mathrm{loc}}=\left\{\left(x, u_{\epsilon}(x)\right): x \in X,\|x\|<\epsilon\right\}
$$

with $u_{\varepsilon}: X \rightarrow Y, u_{\varepsilon}(0)=0, u_{\epsilon}^{\prime}(0)=0,0<\epsilon \leq \epsilon_{0}$. 
We now interpret lemma 3.4 in terms of the original one-step method (1.2) applied to the given differential equation (1.1).

First recall that the number $K$ which is used in the additional cut-off in (3.11) does not depend on $\epsilon$. If $\|\tilde{z}\| \leq K$ then $w(\tilde{z}, h, \epsilon)=\phi\left(\tilde{z}, h, \tilde{f}_{\epsilon}\right)$, thus

$$
\tilde{M}_{\epsilon, h, K}=\left\{\left(\tilde{x}, \tilde{u}_{\epsilon, h}(\tilde{x})\right): \tilde{x} \in X,\|\tilde{x}\| \leq K\right\}
$$

is locally invariant under $\phi\left(\tilde{z}, h, \tilde{f}_{\epsilon}\right)$. (Note that $K \geq 2$ and $\left\|\tilde{u}_{\epsilon, h}(\tilde{z})\right\| \leq 1$, thus a restriction of the $y$-components of $\tilde{M}_{\epsilon, h}$ is superfluous.) Using the scaling invariance (A4) of the flow of the one-step method this implies that

$$
M_{\epsilon, h, K}=\left\{\left(x, u_{\epsilon, h}(x)\right): x \in X,\|x\| \leq \epsilon K\right\}
$$

with

$$
u_{\epsilon, h}(x)=\epsilon \tilde{u}_{\epsilon, h}(x / \epsilon), \quad x \in X
$$

is locally invariant under $\phi\left(z, h, f_{\epsilon}\right)$. If $\|z\| \leq \epsilon / 2$ and $\|z-\zeta\| \leq \epsilon / 2$ then $f_{\epsilon}(\zeta)=f(\zeta)$; therefore, using the locality assumption (A2) of the one-step method, we have

$$
\phi(z, h, f)=\phi\left(z, h, f_{\epsilon}\right) \text { for }\|z\| \leq \epsilon / 2, h \leq \epsilon / 2 c_{2} .
$$

This shows:

Theorem 3.9 The discrete-time flow $z_{j+1}=\phi\left(z_{j}, h, f\right)$ of (1.2) has locally invariant manifolds

$$
M_{\epsilon, h, \mathrm{loc}}=\left\{\left(x, u_{\epsilon, h}(x)\right): x \in X,\|x\| \leq \frac{\epsilon}{2}\right\}
$$

for $0<\epsilon \leq \epsilon_{0}, 0<h \leq \min \left\{h_{0}, c \epsilon\right\}$. Here $u_{h, \epsilon}: X \rightarrow Y$ with $u_{h, \epsilon}(0)=0$. 
Finally, if $\epsilon$ is kept fixed, the manifolds constructed for the one-step method converge towards the local center-unstable manifold of the differential equation. This follows directly from lemma 3.6 and the back-scalings (3.16), (3.17).

Theorem 3.10 For the constructed locally invariant manifolds $M_{\epsilon, \text { loc }}$ of the differential equation and $M_{\epsilon, h, \text { loc }}$ of the one-step method,

$$
\left\|u_{\epsilon}(x)-u_{h, \epsilon}(x)\right\|_{k} \leq c_{\epsilon} h^{p}
$$

where $c_{\epsilon}$ is independent of $h$.

\section{CONSTRUCTION OF THE MANIFOLDS VIA GRAPH} TRANSFORMS.

Henceforth we drop $\sim$ in the notation for the scaled variables and consider a family of flows $z_{j+1}=w\left(z_{j}, h, \epsilon\right)$ where

$$
\begin{aligned}
& w(z, h, \epsilon)=e^{L h} z+\mu(z, h, \epsilon), \\
& z \in \mathbb{R}^{n}, \quad 0<h \leq h_{0}, \quad 0<\epsilon \leq 1
\end{aligned}
$$

and $\mu$ satisfies (3.12). If unimportant, the dependency on $h, \epsilon$ is suppressed notationally. In $(x, y)$-coordinates the function $w$ has the form

$$
\begin{aligned}
& w_{1}(x, y)=e^{A h} x+\mu_{1}(x, y) \in X, \\
& w_{2}(x, y)=e^{B h} y+\mu_{2}(x, y) \in Y .
\end{aligned}
$$

A manifold

$$
M=\{(x, u(x)): x \in X\}, u \in \bar{V}
$$

is invariant for (4.1) if and only if

$$
e^{B h} u(\xi)+\mu_{2}(\xi, u(\xi))=u\left(e^{A h} \xi+\mu_{1}(\xi, u(\xi))\right) \text { for all } \xi \in X
$$

We turn this into the following: For given $x \in X, u \in \bar{V}$ determine

$$
\xi=\xi(x, u, h, \epsilon) \in X
$$


as solution of

$$
e^{A h} \xi+\mu_{1}(\xi, u(\xi))=x
$$

and define

$$
\left(\mathcal{F}_{h, \epsilon} u\right)(x):=e^{B h} u(\xi)+\mu_{2}(\xi, u(\xi))
$$

Then (4.2) requires

$$
\left(\mathcal{F}_{\boldsymbol{h}, \boldsymbol{\epsilon}} \boldsymbol{u}\right)(x)=u(x)
$$

i.e. we ask for a fixed point of $\mathcal{F}=\mathcal{F}_{h, \epsilon}$. Our aim is to show:

Theorem 4.1 Under the above conditions there exist $h_{0}>0, \epsilon_{0}>0$ such that the operators $\mathcal{F}_{h, \epsilon}, 0<h \leq h_{0}, 0<\epsilon \leq \epsilon_{0}$, map $V$ into itself and contract w.r.t. \|\|$_{k}$ with a contraction constant $1-h \kappa, \kappa>$ 0 independent of $h, \epsilon$. Consequently, the operators $\mathcal{F}_{h, \epsilon}$ have unique fixed points $u_{h, \epsilon} \in \bar{V}$.

Proof: 1. We give a detailed proof for $k=0$ and make some remarks towards the general case in the end. In lemma 3.1 choose $N_{1}=4$ and construct \|\|$_{x}$ accordingly. As explained after lemma 3.1 this fixes the norm for $z=(x, y)$; the construction of the cut-off function $\psi$ and the constant $C^{*}$ of (3.12) refer to this specific norm. Then let $\epsilon_{0}>0, h_{0}>0$ be chosen such that

$$
\frac{\beta}{N_{1}} h_{0} \leq \frac{1}{2}, 4 C^{*}\left(\epsilon_{0}+h_{0}\right) \leq \frac{\beta}{2 N_{1}}=\frac{\beta}{8} \leq \frac{1}{8}
$$

Always assume $0<h \leq h_{0}, 0<\epsilon \leq \epsilon_{0}$.

2. Existence and estimates for $\xi=\xi(x)$. Write (4.3) as

$$
\xi=e^{-A h} x-e^{-A h} \mu_{1}(\xi, u(\xi))=: g(\xi)
$$


Lemma 3.1 and (3.12) imply

$$
\begin{aligned}
\| g\left(\xi_{1}\right) & -g\left(\xi_{2}\right)\left\|\leq\left(1+\frac{\beta}{N_{1}} h\right) \cdot\right\| \mu_{1}\left(\xi_{1}, u\left(\xi_{1}\right)\right)-\mu_{1}\left(\xi_{2}, u\left(\xi_{2}\right)\right) \| \\
& \leq \frac{3}{2} C^{*} h(\epsilon+h)\left\{\left\|\xi_{1}-\xi_{2}\right\|+\left\|u\left(\xi_{1}\right)-u\left(\xi_{2}\right)\right\|\right\} \\
& \leq 3 C^{*} h(\epsilon+h)\left\|\xi_{1}-\xi_{2}\right\| \\
& \leq \frac{1}{8} h\left\|\xi_{1}-\xi_{2}\right\| .
\end{aligned}
$$

This shows that $\xi=\xi(x)$ exists uniquely and is a $C^{1}$-function of $x$ for $u \in V$. For $\xi^{\prime}(x)$ obtain from (4.4)

$$
\begin{aligned}
\xi^{\prime}(x) & =e^{-A h}-e^{-A h}\left\{\mu_{1 x}(\cdot)+\mu_{2 y}(\cdot) u^{\prime}(\xi)\right\} \xi^{\prime}(x) \\
& =: e^{-A h}-R \xi^{\prime}(x)
\end{aligned}
$$

Here $\|R\| \leq\left(1+\frac{\beta}{N_{1}} h\right) 2 C^{*} h(\epsilon+h)<\frac{h}{8}$, thus

$$
\xi^{\prime}(x)=(I+R)^{-1} e^{-A h}
$$

This shows that $\xi^{\prime}(x)$ is an $O(h)$-perturbation of the identity. Consequently, $\xi=\xi(x)$ is a 1-1 map onto $\mathbb{R}^{n}$; this ensures that (4.2) holds for all $\xi \in X$ once $u$ is a fixed point under $\mathcal{F}=\mathcal{F}_{h, \epsilon}$. Equation (4.5) also yields the estimate

$$
\begin{aligned}
\left\|\xi^{\prime}(x)\right\| & \leq\left(1+\frac{\beta}{N_{1}} h\right)\left(1-4 C^{*} h(\epsilon+h)\right)^{-1} \\
& \leq\left(1+\frac{\beta}{N_{1}} h\right)\left(1-\frac{\beta h}{2 N_{1}}\right)^{-1} \leq 1+2 \frac{\beta}{N_{1}} h .
\end{aligned}
$$

3. The inclusion $f(V) \subset V$. For $u \in V$ and $x \in X$ :

$$
\begin{aligned}
\|(\mathcal{F} u)(x)\| & \leq\left\|e^{B h}\right\|\|u(\xi)\|+\left\|\mu_{2}(\xi, u(\xi))\right\| \\
& \leq 1-\beta h+C^{*} h(\epsilon+h)<1
\end{aligned}
$$

For the derivative we have

$$
(\mathcal{F} u)^{\prime}(x)=\left\{e^{B h_{u}} u^{\prime}(\xi)+\mu_{2 x}(\cdot)+\mu_{2 y}(\cdot) u^{\prime}(\xi)\right\} \xi^{\prime}(x)
$$


thus

$$
\begin{aligned}
\|(\mathcal{F} u)(x)\| & \leq\left\{1-\beta h+2 C^{*} h(\epsilon+h)\right\}\left(1+2 \frac{\beta}{N_{1}} h\right) \\
& \leq\left(1-\frac{\beta}{2} h\right)\left(1+\frac{\beta}{2} h\right)<1 .
\end{aligned}
$$

Here the choice $N_{1}=4$ was used. Finally, $u(0)=0$ and $\mu_{1}(0,0)=0$ imply $\xi(0)=0$. Thus $(\mathcal{F} u)(0)=\mu_{2}(0,0)=0$, and so $\mathcal{F} u \in V$.

4. The contractivity of $\mathcal{F}$ w.r.t. \|\|$_{0}$.

Let $u_{1}, u_{2} \in V, x \in X$. We first estimate the difference between $\xi_{1}=$ $\xi\left(x, u_{1}, h, \epsilon\right)$ and $\xi_{2}=\xi\left(x, u_{2}, h, \epsilon\right)$. From (4.4)

$$
\xi_{1}-\xi_{2}=-e^{-A h}\left\{\mu_{1}\left(\xi_{1}, u_{1}\left(\xi_{1}\right)\right)-\mu_{1}\left(\xi_{2}, u_{2}\left(\xi_{2}\right)\right)\right\},
$$

thus

$$
\begin{aligned}
\left\|\xi_{1}-\xi_{2}\right\| \leq\left(1+\frac{\beta}{N_{1}} h\right) C^{*} h(\epsilon+h)\left(\left\|\xi_{1}-\xi_{2}\right\|+\left\|u_{1}\left(\xi_{1}\right)-u_{1}\left(\xi_{2}\right)\right\|\right. \\
\left.+\left\|u_{1}\left(\xi_{2}\right)-u_{2}\left(\xi_{2}\right)\right\|\right) \\
\leq \frac{3}{2} C^{*} h(\epsilon+h)\left\{2\left\|\xi_{1}-\xi_{2}\right\|+\left\|u_{1}-u_{2}\right\|_{0}\right\}
\end{aligned}
$$

and so

$$
\begin{aligned}
\left\|\xi_{1}-\xi_{2}\right\| & \leq \frac{\frac{3}{2}}{1-3 C^{*} h(\epsilon+h)} C^{*} h(\epsilon+h)\left\|u_{1}-u_{2}\right\|_{0} \\
& \leq 2 C^{*} h(\epsilon+h)\left\|u_{1}-u_{2}\right\|_{0} .
\end{aligned}
$$

This yields the following estimate for $\mathcal{F} u_{1}-\mathcal{F} u_{2}$ :

$$
\begin{aligned}
& \left\|\left(\mathcal{F} u_{1}\right)(x)-\left(\mathcal{F} u_{2}\right)(x)\right\| \leq\left\|e^{B h}\right\|\left\|u_{1}\left(\xi_{1}\right)-u_{2}\left(\xi_{2}\right)\right\| \\
& \quad+\left\|\mu_{2}\left(\xi_{1}, u_{1}\left(\xi_{1}\right)\right)-\mu_{2}\left(\xi_{2}, u_{2}\left(\xi_{2}\right)\right)\right\| \\
& \quad \leq(1-\beta h)\left\{\left\|\xi_{1}-\xi_{2}\right\|+\left\|u_{1}-u_{2}\right\|_{0}\right\}+C^{*} h(\epsilon+h)\left\{2\left\|\xi_{1}-\xi_{2}\right\|+\left\|u_{1}-u_{2}\right\|_{0}\right\} \\
& \quad \leq\left(1-\beta h+2 C^{*} h(\epsilon+h)\right)\left\{\left\|\xi_{1}-\xi_{2}\right\|+\left\|u_{1}-u_{2}\right\|_{0}\right\} \\
& \quad \leq\left(1-\beta h+2 C^{*} h(\epsilon+h)\right)\left(1+2 C^{*} h(\epsilon+h)\right)\left\|u_{1}-u_{2}\right\|_{0} .
\end{aligned}
$$

The coefficient of $\left\|u_{1}-u_{2}\right\|_{0}$ can be estimated by 


$$
\left(1-\beta h+\frac{1}{16} \beta h\right)\left(1+\frac{1}{16} \beta h\right) \leq 1-\frac{7}{8} \beta h,
$$

which finishes the proof of the contractivity.

5. Treatment of higher derivatives Consider the case of a general integer $k$ and recall for $u \in V$ the estimates

$$
\left\|\frac{\partial^{|\alpha|}}{\partial x^{\alpha}} u(x)\right\| \leq 1, \quad x \in X,|\alpha| \leq k+1 .
$$

Also, recall the estimates (3.12) for $\mu$. The function $\xi=\xi(x)$ clearly has $k+1$ derivatives and for $2 \leq|\alpha| \leq k+1$ one shows

$$
\left\|\frac{\partial^{|\alpha|}}{\partial x^{\alpha}} \xi(x)\right\| \leq c_{1} h(\epsilon+h)
$$

where $c_{1}$ depends only on $C^{*}$ and $k$. To prove the inclusion $\mathcal{f}(V) \subset$ $V$ one has to differentiate

$$
(\mathcal{F} u)(x)=e^{B h} u(\xi)+\mu_{2}(\xi, u(\xi))
$$

repeatedly with respect to $x$-variables. One finds for $|\alpha| \leq k+1$ :

$$
\left\|\frac{\partial^{|\alpha|}}{\partial x^{\alpha}}(\mathcal{F} u)(x)\right\| \leq(1-\beta h)\left(1+2 \frac{\beta}{N_{1}} h\right)^{k+1}+c_{2} h(\epsilon+h)
$$

where $c_{2}$ depends only on $C^{*}$ and $k$. If $N_{1}, h_{0}, \epsilon_{0}$ are chosen appropriately we find $\left\|\mathcal{F}_{h, \epsilon} u\right\|_{k+1} \leq 1$. To prove contractivity of $\mathcal{F}$ w.r.t. \|\|$k$ differentiate the identity

$$
\begin{gathered}
\left(\mathcal{F} u_{1}\right)(x)-\left(\mathcal{F} u_{2}\right)(x)=e^{B h}\left\{u_{1}\left(\xi_{1}\right)-u_{2}\left(\xi_{2}\right)\right\} \\
+\mu_{2}\left(\xi_{1}, u_{1}\left(\xi_{1}\right)\right)-\mu_{2}\left(\xi_{2}, u_{2}\left(\xi_{2}\right)\right)
\end{gathered}
$$

w.r.t. $x$-variables. Similarly we find for $|\alpha| \leq k$ :

$$
\begin{aligned}
& \left\|\frac{\partial^{|\alpha|}}{\partial x^{\alpha}}\left\{\left(\mathcal{F} u_{1}\right)(x)-\left(\mathcal{F} u_{2}\right)(x)\right\}\right\| \\
& \quad \leq\left(1-\beta h+c_{3} h(\epsilon+h)\right)\left(1+c_{4} h(\epsilon+h)\right)\left\|u_{1}-u_{2}\right\|_{k} \\
& \quad \leq\left(1-\frac{\beta}{2} h\right)\left\|u_{1}-u_{2}\right\|_{k}
\end{aligned}
$$

for $0<h \leq h_{0}, 0<\epsilon \leq \epsilon_{0}$, if $h_{0}, \epsilon_{0}$ are sufficiently small.

This finishes the proof of Theorem 4.1 and also of lemma 3.4. 


\section{THE MANIFOLDS FOR THE FLOW OF THE DIFFERENTIAL} EQUATION.

According to (3.13) the result of Theorem 4.1 applies to the $h$-flows of the scaled cut-off differential equations (3.2). Again, we drop the $\sim$ notation for the scaled variables. Let $G_{h, \epsilon}: \bar{V} \rightarrow \bar{V}$ denote the operator corresponding to the flow (3.13):

$$
\Phi\left(z, h, \tilde{f}_{\epsilon}\right)=e^{L h} z+\rho(z, h, \epsilon) .
$$

Henceforth fix $\epsilon \leq \epsilon_{0}$ and write $\Phi$ in the $(x, y)$ - coordinate system:

$$
\begin{aligned}
& \Phi_{1}(x, y, h)=e^{A h} x+\rho_{1}(x, y, h) \in X, \\
& \Phi_{2}(x, y, h)=e^{B h} y+\rho_{2}(x, y, h) \in Y .
\end{aligned}
$$

The operator $G_{h}=G_{h, \epsilon}$ is defined by

$$
\left(G_{h} u\right)(x)=\Phi_{2}(\zeta, u(\zeta), h)
$$

where $\zeta=\zeta(x, u, h) \in X$ is the unique solution of

$$
\Phi_{1}(\zeta, u(\zeta), h)=x
$$

In other words, the operator $G_{h}$ is determined by the flow $z \rightarrow \phi\left(z, h, \tilde{f}_{\epsilon}\right)$ in exactly the same way as the operator $F_{h, \epsilon}$ is determined by the flow $z \rightarrow$ $w(z, h, \epsilon)$. According to Theorem 4.1 the operators $G_{h}$ have unique fixed points $u_{h} \in \bar{V}$ for $h \leq h_{0}$. We first show:

\section{Lemma 5.1}

If $h+h^{\prime} \leq h_{0}$ then

$$
G_{h} \circ G_{h^{\prime}}=G_{h+h^{\prime}}=G_{h^{\prime}} \circ G_{h}
$$

and consequently $u_{h}=u_{h^{\prime}}$.

\section{Proof:}

1. Let $u \in \bar{V}$ and $x \in X$ be given, and let $\zeta \in X$ solve

$$
\Phi_{1}\left(\zeta, u(\zeta), h+h^{\prime}\right)=x \text {. }
$$


Then

$$
\begin{aligned}
& \left(G_{h+h^{\prime}} u\right)(x)=\Phi_{2}\left(\zeta, u(\zeta), h+h^{\prime}\right) \\
& =\Phi_{2}\left(\Phi_{1}\left(\zeta, u(\varsigma), h^{\prime}\right), \Phi_{2}\left(\varsigma, u(\varsigma), h^{\prime}\right), h\right) .
\end{aligned}
$$

The latter equality is due to the semigroup property of the flows. Now let

$$
\tilde{\zeta}:=\Phi_{1}\left(s, u(\zeta), h^{\prime}\right), \quad v:=G_{h^{\prime}} u
$$

By the definition of $G_{h^{\prime}} u$ we have

$$
v(\tilde{\zeta})=\left(G_{h^{\prime}} u\right)(\xi)=\Phi_{2}\left(\zeta, u(\zeta), h^{\prime}\right)
$$

Using the semigroup property again yields

$$
\begin{aligned}
x & =\Phi_{1}\left(\zeta, u(\zeta), h+h^{\prime}\right) \\
& =\Phi_{1}\left(\Phi_{1}\left(\zeta, u(\zeta), h^{\prime}\right), \Phi_{2}\left(\zeta, u(\zeta), h^{\prime}\right), h\right) \\
& =\Phi_{1}(\tilde{\zeta}, v(\tilde{\zeta}), h) .
\end{aligned}
$$

This identity implies

$$
\left(G_{h} v\right)(x)=\Phi_{2}(\tilde{\zeta}, v(\tilde{\zeta}), h)
$$

by the definition of $G_{h} v$. Summarizing:

$$
\begin{aligned}
\left\{\left(G_{h} \circ G_{h^{\prime}}\right) u\right\}(x)=\left\{G_{h} v\right\}(x) & \\
& =\Phi_{2}(\tilde{\zeta}, v(\tilde{\zeta}), h) \\
& =\Phi_{2}\left(\Phi_{1}\left(\zeta, u(\zeta), h^{\prime}\right), \Phi_{2}\left(\zeta, u(\zeta), h^{\prime}\right), h\right) \text { by }(5.2) \\
& =\left\{G_{h+h^{\prime}} u\right\}(x) \text { by }(5.1) .
\end{aligned}
$$

Thus $G_{h} \circ G_{h^{\prime}}=G_{h^{\prime}} \circ G_{h}$ is proved.

2. The function $u_{h}$ is a fixed point of $G_{h}$. This implies

$$
G_{h^{\prime}} u_{h}=\left(G_{h^{\prime}} \circ G_{h}\right) u_{h}=\left(G_{h} \circ G_{h^{\prime}}\right) u_{h}=G_{h}\left(G_{h^{\prime}} u_{h}\right)
$$

Using uniqueness of the fixed point of $G_{h}$ it follows that

$$
G_{h^{\prime}} u_{h}=u_{h}
$$


Then uniqueness of the fixed point of $G_{h^{\prime}}$ yields $u_{h}=u_{h^{\prime}}$.

As a consequence of lemma 5.1 the fixed point $u_{h, \epsilon}$ of $G_{h, \varepsilon}$ is independent of $h$ for $0<h \leq h_{1}=h_{0} / 2,0<\epsilon \leq \epsilon_{0}$, and we write $u_{\epsilon}=u_{h, \epsilon}$. The manifold

$$
M_{\epsilon}=\left\{\left(x, u_{\epsilon}(x)\right): x \in X\right\}
$$

is globally invariant under the discrete flows

$$
z_{j+1}=\Phi\left(z_{j}, h, \tilde{f}_{\epsilon}\right) \text { for } 0<h \leq h_{1}
$$

Again, the semigroup property implies invariance of $M_{\epsilon}$ under the continuous-time flow

$$
\Phi\left(z, t, \tilde{f}_{\epsilon}\right), \quad t \geq 0
$$

In order to finish the proof of lemma 3.5 it remains to show that $u_{\epsilon}^{\prime}(0)=0$ for $k \geq 1$. To this end take an arbitrary $u \in V$ with $u^{\prime}(0)=0$ and let $v=G_{h} u$. From the defining equation for $G_{h}$ it follows that:

$$
v(x)=e^{B h} u(\zeta)+\rho_{2}(\zeta, u(\zeta), h), \zeta=\zeta(x)
$$

and thus

$$
v^{\prime}(0)=e^{B h} u^{\prime}(\varsigma(0)) \varsigma^{\prime}(0)=e^{B h} u^{\prime}(0) \varsigma^{\prime}(0)=0
$$

Therefore all functions in the sequence of iterates $G_{h}^{\nu} u, \nu=1,2, \ldots$ have the derivative 0 at $x=0$. Since the sequence converges to the limit $u_{\epsilon}$ w.r.t. \|\|$_{k}$ the assertion follows.

\section{ERROR ESTIMATES FOR $h \rightarrow 0$.}

In this section let $u_{h, \epsilon}$ denote the fixed point of $\mathcal{F}_{h, \epsilon}$ where $\mathcal{F}_{h, \epsilon}$ is the operator corresponding to the flows $w(z, h, \epsilon)$ of the modified onestep method, see section 4; furthermore, let $u_{\epsilon}$ denote the fixed point of $G_{h, \epsilon}$ where $G_{h, \epsilon}$ corresponds to the flow $\Phi\left(z, h, \tilde{f}_{\epsilon}\right)$ of the scaled cut-off differential equation (3.2), see section 5. We want to estimate

$$
\left\|u_{\epsilon}-u_{h, \epsilon}\right\|_{k}
$$


using the relation (3.14) between the flows $w$ and $\Phi$. Recall from Theorem 4.1 the contractivity

$$
\left\|\mathcal{F}_{h, \epsilon} u-\mathcal{F}_{h, \epsilon} v\right\|_{k} \leq(1-h \kappa)\|u-v\|_{k}
$$

which implies

$$
\begin{aligned}
& \left\|u-\mathcal{F}_{h, \epsilon} u-\left(v-\mathcal{F}_{h, \epsilon} v\right)\right\|_{k} \\
& \quad \geq\|u-v\|_{k}-\left\|\mathcal{F}_{h, \epsilon} u-\mathcal{F}_{h, \epsilon} v\right\|_{k} \geq h \kappa\|u-v\|_{k},
\end{aligned}
$$

i.e. $\|u-v\|_{k} \leq \frac{1}{h \kappa}\left\|u-\mathcal{F}_{h, \epsilon} u-\left(v-\mathcal{F}_{h, \epsilon} v\right)\right\|_{k}$. Then in particular

$$
\begin{aligned}
\left\|u_{\epsilon}-u_{h, \epsilon}\right\|_{k} & \leq \frac{1}{h \kappa}\left\|u_{\epsilon}-\mathcal{F}_{h, \epsilon} u_{\epsilon}\right\|_{k} \\
& =\frac{1}{h \kappa}\left\|G_{h, \epsilon} u_{\epsilon}-\mathcal{F}_{h, \epsilon} u_{\epsilon}\right\|_{k} .
\end{aligned}
$$

We first estimate the right side for $k=0$. Let $x \in X$ be fixed, and let $\xi$ and $\varsigma \in X$ be determined by

$$
w_{1}\left(\xi, u_{\epsilon}(\xi)\right)=x \text { and } \Phi_{1}\left(\zeta, u_{\epsilon}(\zeta)\right)=x,
$$

respectively. Then by definition

$$
\begin{aligned}
\left(G_{h, \epsilon} u_{\epsilon}\right)(x)-\left(\mathcal{F}_{h, \epsilon} u_{\epsilon}\right)(x) \\
=\Phi_{2}\left(\zeta, u_{\epsilon}(\zeta)\right)-w_{2}\left(\xi, u_{\epsilon}(\xi)\right) \\
=\Phi_{2}\left(\zeta, u_{\epsilon}(\zeta)\right)-\Phi_{2}\left(\xi, u_{\epsilon}(\xi)\right) \\
+\Phi_{2}\left(\xi, u_{\epsilon}(\xi)\right)-w_{2}\left(\xi, u_{\epsilon}(\xi)\right),
\end{aligned}
$$

thus

$$
\begin{gathered}
\left\|\left(G_{h, \epsilon} u_{\epsilon}\right)(x)-\left(\mathcal{F}_{h, \epsilon} u_{\epsilon}\right)(x)\right\| \\
\leq c_{1}\|\zeta-\xi\|+c_{2} h^{p+1}
\end{gathered}
$$

using the uniform boundedness of the first derivatives of $\Phi$ and the estimate (3.14) for $\alpha=0$. It remains to estimate $\|\xi-\xi\|$. Note

$$
\begin{aligned}
& w_{1}\left(\zeta, u_{\epsilon}(\zeta)\right)-w_{1}\left(\xi, u_{\epsilon}(\xi)\right) \\
& \quad=\Phi_{1}\left(\zeta, u_{\epsilon}(\zeta)\right)+O\left(h^{p+1}\right)-w_{1}\left(\xi, u_{\epsilon}(\xi)\right) \\
& \quad=x+O\left(h^{p+1}\right)-x=O\left(h^{p+1}\right),
\end{aligned}
$$


where the $O\left(h^{p+1}\right)$-term results from (3.14). In part 2 of the proof of Theorem 4.1 we showed unique solvability of the solution $\xi$ of

$$
x=w_{1}\left(\xi, u_{\epsilon}(\xi)\right)=e^{A h} \xi+\mu_{1}\left(\xi, u_{\epsilon}(\xi)\right)
$$

The contraction argument given there clearly reveals

$$
\|s-\xi\| \leq c_{3} h^{p+1}
$$

as a consequence of

$$
\left\|w_{1}\left(\zeta, u_{\epsilon}(\zeta)\right)-w_{2}\left(\xi, u_{c}(\xi)\right)\right\| \leq c_{4} h^{p+1}
$$

This finishes the proof for $k=0$, i.e. the estimate $\left\|u_{\epsilon}-u_{h, \epsilon}\right\|_{0} \leq C h^{p}$.

To obtain the result for general $k$ it suffices to show

$$
\left\|G_{h, \epsilon} u_{\epsilon}-\mathcal{F}_{h, \epsilon} u_{\epsilon}\right\|_{k} \leq C h^{p+1}
$$

because of (6.1). To this end apply the operator

$$
\frac{\partial^{|\alpha|}}{\partial x^{\alpha}},|\alpha| \leq k
$$

to the identity (6.2). Using the uniform bounds for the derivatives of $\Phi_{2}$ and of $u_{\epsilon}$ and using estimate (3.14) it clearly is sufficient to prove

$$
\|\zeta-\xi\|_{k} \leq c_{5} h^{p+1}
$$

But this follows from

$$
w_{1}\left(\xi, u_{\varepsilon}(\xi)\right)=\Phi_{1}\left(\zeta, u_{\epsilon}(\zeta)\right)
$$

and (3.14). This finishes the proof of lemma 3.6.

\section{PROPERTES OF THE MANIFOLDS OF THE ONE-STEP METHOD}

One of the main features of the center-unstable manifolds $M_{e, \text { loc }}$ for the differential equation (1.1) is their property of being attracting at an exponential rate. This is well-known and plausible: The influence of all stable eigenvalues $\lambda$ of $f^{\prime}(0)$, i.e. eigenvalues with $R e \lambda<0$, can be separated 
from the influence of the other eigenvalues. The dynamical behaviour inside $M_{\epsilon, \text { loc }}$ reflects the influence of those eigenvalues $\lambda$ with $\operatorname{Re} \lambda \geq 0$ whereas the stable eigenvalues lead to exponential attraction towards $M_{\epsilon, \text { loc }}$.

In this section we show that the property of being exponentially attracting also holds for the constructed invariant manifolds $M_{\epsilon, h \text {, loc }}$ of the discrete flows (1.2). This holds true whether or not $M_{\epsilon, h, \text { loc }}$ is the center-unstable manifold of the discrete flow. To obtain a clearer understanding of this fact and of the role which the implication (1.6) plays let us first define the term 'growth function' of a one-step method.

Definition: If the method (1.2) applied to (1.1) with an arbitrary linear function $f(z)=L z$ can be written as

$$
z_{j+1}=\phi\left(z_{j}, h, L\right) \equiv \gamma(h L) z_{j}, \quad j=0,1, \ldots
$$

then $\gamma$ is called the 'growth function' of the method. Here $\gamma$ is a complex valued analytic function defined in the neighborhood of the origin in $\mathbf{C}$. (The function $\gamma$ is naturally defined also for all arguments $h L$, where $L$ is a square matrix and $h$ is sufficiently small.)

Simple examples are:

$$
\begin{aligned}
& \gamma_{1}(h \lambda)=1+h \lambda \text { for the explicit Euler method, } \\
& \gamma_{2}(h \lambda)=\frac{1}{1-h \lambda} \text { for the implicit Euler method, } \\
& \gamma_{3}(h \lambda)=\frac{2+h \lambda}{2-h \lambda} \text { for the trapezoidal rule. }
\end{aligned}
$$

For all commonly used one-step methods a growth function is well defined. Furthermore, in addition to the conditions (A0) to (A4) of section 2 assume:

$\left(\right.$ A2 $\left.^{*}\right)$ For each $c_{1}$ there exist $c_{2}, c_{3}, h_{0}$ such that

$$
\|\phi(z, h, f)-\phi(z, h, g)\| \leq c_{3} \sup \left\{\|f(\zeta)-g(\varsigma)\|,\|z-\varsigma\| \leq c_{2} h\right\}
$$

whenever $\|z\| \leq c_{1}$ and $0 \leq h \leq h_{0}$.

This condition is slightly more restrictive than (A2), but it is also satisfied for all commonly used methods. We note that (AO), (A4), and $\left(\mathrm{A}^{*}\right.$ ) imply 


$$
\frac{\partial \phi}{\partial z}(0, h, f)=\gamma\left(h f^{\prime}(0)\right)
$$

This is important, since the local behaviour of the flow of the one-step method near the origin $z=0$ is governed by its linearization $\frac{\partial \phi}{\partial z}(0, h, f)$, and thus by the growth function $\gamma$ of the method. To prove (7.1) first note:

$$
\begin{aligned}
& \left\|\phi\left(z, h, f_{\epsilon}\right)-\phi\left(z, h, f^{\prime}(0)\right)\right\| \\
& \quad \leq c_{3} \sup \left\{\left\|\frac{1}{\epsilon} f(\epsilon \zeta)-f^{\prime}(0) \zeta\right\|,\|z-\zeta\| \leq c_{2} h\right\} \rightarrow 0 \text { for } \epsilon \rightarrow 0
\end{aligned}
$$

by $\left(\mathrm{A2}^{*}\right)$ and (A4). Furthermore, we have

$$
\begin{gathered}
\phi\left(z, h, f_{\epsilon}\right)=\frac{1}{\epsilon} \phi(\epsilon z, h, f)=\frac{1}{\epsilon}\{\phi(\epsilon z, h, f)-\phi(0, h, f)\} \\
\rightarrow \frac{\partial \phi}{\partial z}(0, h, f) z \text { for } \epsilon \rightarrow 0
\end{gathered}
$$

and

$$
\phi\left(z, h, f^{\prime}(0)\right)=\gamma\left(h f^{\prime}(0)\right) z .
$$

Thus equation (7.1) follows.

Let us point out relevant implications of (7.1). From our consistency assumption for the one-step method it follows that $\gamma(h \lambda)=\exp (h \lambda)+$ $O\left(h^{p+1}\right)$ and thus

$$
\begin{aligned}
& \operatorname{Re} \lambda<0 \text { implies }|\gamma(h \lambda)|<1 \text { for } h \leq h_{0}(\lambda), \\
& \operatorname{Re} \lambda>0 \text { implies }|\gamma(h \lambda)|>1 \text { for } h \leq h_{0}(\lambda) .
\end{aligned}
$$

For this reason, if $L=f^{\prime}(0)$ has no eigenvalue $\lambda$ with $\operatorname{Re} \lambda=0$, then the stability characteristics of the flows near $z=0$ are exactly the same for the differential equation flow and for the discrete flow of the one-step method. This follows from (7.1) and (7.2). If there are eigenvalues $\lambda$ of $L=f^{\prime}(0)$ with $\operatorname{Re} \lambda=0$, then the situation is more complicated. For example, assume there is an eigenvalue $\lambda \neq 0$ with $\operatorname{Re} \lambda=0$. Then

$$
\begin{aligned}
& \left|\gamma_{1}(h \lambda)\right|=|1+h \lambda|>1, \\
& \left|\gamma_{2}(h \lambda)\right|=\frac{1}{|1-h \lambda|}<1, \\
& \left|\gamma_{3}(h \lambda)\right|=\left|\frac{2+h \lambda}{2-h \lambda}\right|=1
\end{aligned}
$$


for the three growth functions mentioned above. For the differential equation flow the eigenvalue $\lambda$ contributes to the center space. For the discrete flows, however, the corresponding eigenvalue

$$
\gamma_{i}(h \lambda), \quad i=1,2,3
$$

of the linear operator (7.1) contributes to: the unstable space for $i=1$ (explicit Euler), the stable space for $i=2$ (implicit Euler), the center space for $i=3$ (trapezoidal rule). The constructed invariant manifolds $M_{\epsilon, h, \text { loc }}$ for the discrete flows always approximate the center-unstable manifolds $M_{\epsilon \text {,loc }}$ of the differential equation. Nevertheless, as the example of the implicit Euler method shows, $M_{\epsilon, h \text {,loc }}$ will, in general, contain contributions of eigenvalues $\gamma(h \lambda)$ which are stable for the discrete flows. This explains why $M_{\epsilon, h, l o c}$ is, in general, not the center-unstable manifold for discrete flows. However, because of the implication (7.2a) it is plausible - and indeed true - that $M_{\epsilon, h, \text { loc }}$ attracts trajectories at an exponential rate. For technical reasons we consider first the modified discrete flows $w(z, h, \epsilon)$ defined in (3.11) for which the following global result holds:

Lemma 7.1

Let $\left(x_{0}, y_{0}\right) \in X \oplus Y$ denote a starting point with $\left\|y_{0}\right\| \leq \frac{1}{4}$ and consider the iterates

$$
\left(x_{j+1}, y_{j+1}\right)=w\left(x_{j}, y_{j}, h, \epsilon\right), j=0,1, \ldots
$$

Then

$$
\left\|y_{j}-u_{h, \epsilon}\left(x_{j}\right)\right\| \leq e^{-\kappa j h}\left\|y_{0}-u_{h, \epsilon}\left(x_{0}\right)\right\|
$$

for $j=0,1, \ldots$. Here $\kappa>0$ is the constant governing the contraction ratio in Theorem 4.1.

Proof:

From part 3 of the proof of Theorem 4.1 it is easy to see that the operator $\mathcal{F}=\mathcal{F}_{h, \epsilon}$ actually maps the set

$$
\left\{u \in V:\|u\|_{0} \leq \frac{1}{4}\right\}
$$

into itself. (Possibly $h_{0}$ and $\epsilon_{0}$ have to be chosen smaller.) Thus we can assume

$$
\left\|u_{h, \epsilon}\right\|_{0} \leq \frac{1}{4}
$$


Now consider the functions

$$
v_{j}(x)=u_{h, \epsilon}(x)+y_{j}-u_{h, \epsilon}\left(x_{j}\right), \quad x \in X, \quad j=0,1, \ldots
$$

which differ from $u_{h, \epsilon}(x)$ by constants depending on $j$. If $\left\|y_{j}-u_{h, \epsilon}\left(x_{j}\right)\right\| \leq$ $\frac{1}{2}$ then $\left\|v_{j}\right\|_{0} \leq\left\|u_{h, \epsilon}\right\|_{0}+\frac{1}{2} \leq \frac{3}{4}$, and therefore $v_{j} \in V$. The contractivity of $₹$ yields

$$
\begin{aligned}
& \left\|\mathcal{F} v_{j}-u_{h, \epsilon}\right\|_{k}=\left\|\mathcal{F} v_{j}-\mathcal{F} u_{h, \epsilon}\right\|_{k} \\
& \quad \leq(1-\kappa h)\left\|v_{j}-u_{h, \epsilon}\right\|_{k}=(1-\kappa h)\left\|y_{j}-u_{h, \epsilon}\left(x_{j}\right)\right\|
\end{aligned}
$$

Clearly

$$
x_{j+1}=w_{1}\left(x_{j}, y_{j}\right)=w_{1}\left(x_{j}, v_{j}\left(x_{j}\right)\right)
$$

and

$$
y_{j+1}=w_{2}\left(x_{j}, y_{j}\right)=w_{2}\left(x_{j}, v_{j}\left(x_{j}\right)\right)
$$

implies $\left(f v_{j}\right)\left(x_{j+1}\right)=y_{j+1}$; thus we get

$$
\begin{aligned}
\left\|y_{j+1}-u_{h, \epsilon}\left(x_{j+1}\right)\right\| & \leq(1-\kappa h)\left\|y_{j}-u_{h, \epsilon}\left(x_{j}\right)\right\| \\
& \leq e^{-\kappa h}\left\|y_{j}-u_{h, \epsilon}\left(x_{j}\right)\right\| .
\end{aligned}
$$

The proof of the lemma now follows by induction on $j$.

Again, this result proved for the modified flows can be reinterpreted in terms of the original one-step method. To this end compare the remarks preceding Theorem 3.9. One obtains:

\section{Theorem 7.2}

Let $\epsilon>0$ and $h>0$ denote sufficiently small numbers for which the manifold

$$
M_{\epsilon, h, \text { loc }}=\left\{\left(x, u_{h, \epsilon}(x)\right): x \in X,\|x\|<\frac{\epsilon}{2}\right\}
$$

constructed in Theorem 3.9 is locally invariant. Let $\left(x_{0}, y_{0}\right)$ denote a starting point with $\left\|y_{0}\right\| \leq \frac{\epsilon}{4}$ and assume the iterates

$$
\left(x_{j+1}, y_{j+1}\right)=\phi\left(x_{j}, y_{j}, h, f\right), j=0,1, \ldots
$$

stay in $B_{\epsilon / 2}(0)$ for $j=0,1, \ldots, J$. Then

$$
\left\|y_{j}-u_{h, \epsilon}\left(x_{j}\right)\right\| \leq e^{-\gamma j h}\left\|y_{0}-u_{h, \epsilon}\left(x_{0}\right)\right\| \text { for } j=0,1, \ldots, J \text {. }
$$


Here $\gamma>0$ is the number governing the contraction in Theorem 4.1, thus $\gamma$ is independent of $\epsilon$ and $h$.

We want to apply this theorem to sharpen - under special assumptions - a recent result of Kloeden and Lorenz [4] about attracting sets $\Lambda$ for differential equations and corresponding sets $\Lambda(h)$ for one-step methods. To illustrate the result in [4] first consider two simple examples which can be analyzed explicitly.

Example 1 (compare [2])

Transform the equation

$$
d r / d t=-r^{3}, \quad d \alpha / d t=1
$$

from polar coordinates $(r, \alpha)$ to Cartesian coordinates

$$
\xi=r \sin \alpha, \quad \eta=r \cos \alpha
$$

to obtain

$$
\begin{aligned}
& d \xi / d t=\eta-\xi\left(\xi^{2}+\eta^{2}\right)=: f_{1}(\xi, \eta), \\
& d \eta / d t=-\xi-\eta\left(\xi^{2}+\eta^{2}\right)=: f_{2}(\xi, \eta) .
\end{aligned}
$$

The point $z=(\xi, \eta)=0$ is asymptotically stable; the matrix $f^{\prime}(0)$ has the eigenvalues $\pm i$. Now discretize (7.3) by the explicit Euler method with fixed (small) step-size $h$ :

$$
\begin{aligned}
& \xi_{j+1}=\xi_{j}+h\left\{\eta_{j}-\xi_{j}\left(\xi_{j}^{2}+\eta_{j}^{2}\right)\right\}=: \phi_{1}\left(\xi_{j}, \eta_{j}, h\right), \\
& \eta_{j+1}=\eta_{j}+h\left\{-\xi_{j}-\eta_{j}\left(\xi_{j}^{2}+\eta_{j}^{2}\right)\right\}=: \phi_{2}\left(\xi_{j}, \eta_{j}, h\right) .
\end{aligned}
$$

Here $\frac{\partial \phi}{\partial z}(0,0, h)=\left(\begin{array}{cc}1 & h \\ -h & 1\end{array}\right)$ has the eigenvalues $1 \pm i h$ which lie outside the unit circle. Consequently $z=0$ is an unstable fixed point for (7.4) for each $h$. The general result of [4] shows, however, that the attracting set $\Lambda=\{0\}$ of (7.3) leads to nearby attracting sets $\Lambda(h)$ for the discretization; in general, the Hausdorff distance between $\Lambda(h)$ and $\Lambda$ tends to zero with $h \rightarrow 0$. For (7.4) such a set $\Lambda(h)$ can easily be constructed without following the general approach of [4]. If we define

$$
R_{j}=r_{j}^{2}=\xi_{j}^{2}+\eta_{j}^{2}, \quad j=0,1, \ldots
$$

then a simple computation shows 


$$
R_{j+1}=\left(1+h^{2}\right) R_{j}-2 h R_{j}^{2}+h^{2} R_{j}^{3}
$$

In particular the flow (7.4) maps the circle

$$
\left\{(\xi, \eta): \xi^{2}+\eta^{2}=R\right\}
$$

into the circle

$$
\left\{(\xi, \eta): \xi^{2}+\eta^{2}=F(R)\right\}
$$

with $F(R)=\left(1+h^{2}\right) R-2 h R^{2}+h^{2} R^{3}$. The function $F(R), R \geq 0$, has three fixed points:

$$
\begin{aligned}
& R^{(0)}=0 \\
& R^{(1)}=\frac{1}{h}-\left(\frac{1}{h^{2}}-1\right)^{1 / 2}=\frac{h}{2}+O\left(h^{3}\right) \\
& R^{(2)}=\frac{1}{h}-\left(\frac{1}{h^{2}}-1\right)^{1 / 2}=\frac{2}{h}+O(1)
\end{aligned}
$$

The fixed point $R^{(0)}=0$ is unstable for all $h>0$ in agreement with the instability of $z=(0,0)$ for (7.4). The fixed point $R^{(1)}$ is stable; this leads to the attracting set

$$
\Lambda(h)=\left\{(\xi, \eta): \xi^{2}+\eta^{2} \leq R^{(1)}\right\}
$$

for (7.4) with radius $\sim \sqrt{h}$.

If we apply the theory presented in this paper to Example 1 we do not obtain any information since the center-unstable space $X$ equals the whole space $\mathbf{R}^{2}$. Now adjoin to (7.3) a (trivial uncoupled) decaying part to get:

\section{Example 2}

$$
\begin{aligned}
& d \xi / d t=\eta-\xi\left(\xi^{2}+\eta^{2}\right) \\
& d \eta / d t=-\xi-\eta\left(\xi^{2}+\eta^{2}\right) \\
& d \xi / d t=-\xi
\end{aligned}
$$

Here

$$
\begin{aligned}
& X=\{(\xi, \eta, 0): \xi, \eta \in \mathbb{R}\}, \\
& Y=\{(0,0, \zeta): \varsigma \dot{\mathbb{R}}\} .
\end{aligned}
$$

Again, $z=(0,0,0)$ is asymptotically stable. 
The general theory of [4] applies to the attracting set $\Lambda=\{0\}$; if

$$
z_{j+1}=\phi\left(z_{j}, h\right), j=0,1, \ldots
$$

is a flow of a one-step method then, for $h$ sufficiently small, there is an attracting set $\Lambda(h)$ for this flow. $(\Lambda(h)$ is, in general, not unique.) The following properties of $\Lambda(h)$ can be obtained from the general theory:

(P0) $\Lambda(h)$ contains $\Lambda=\{0\}$ in its interior.

(P1) $\Lambda(h)$ is compact and positively invariant under (7.6).

(P2) $\Lambda(h)$ 'absorbs' nearby trajectories in finite time $T(h)$; more precisely, there exists an open set $U_{0}$ independent of $h, \Lambda(h) \subset U_{0} \subset \mathbb{R}^{n}$, and for each $h \leq h_{0}$ there is a time $T(h)$ such that $z_{0} \in U_{0}$ and $h j \geq T(h)$ imply $z_{j} \in \Lambda(h)$.

(P3) The Hausdorff distance between $\Delta(h)$ and $\Lambda$ tends to zero as $h \rightarrow 0$; in the present situation this just says that the diameter of $\Lambda(h)$ shrinks to zero as $h \rightarrow 0$.

Remark: The sets (7.5) for Example 1 have the properties (P0), (P1) and (P3), but not (P2). If we increase $R^{(1)}$ by $o(h)$ then also (P2) is satisfied.

A set $\Lambda(h)$ with properties (P0) to (P3) for Example 2 and Euler's method is

$$
\Lambda(h)=\left\{(\xi, \eta, \zeta): \xi^{2}+\eta^{2} \leq R^{(1)}+o(h),|\zeta| \leq o(h)\right\} .
$$

If we neglect - for fixed $h$ - the $o(h)$-terms we obtain the set

$$
\Lambda_{c}(h)=\left\{(\xi, \eta, 0): \xi^{2}+\eta^{2} \leq R^{(1)}\right\}
$$

with properties (P1), (P3) and, instead of (P2),

(P2) $\Lambda_{c}(h)$ attracts nearby trajectories, i.e. if $z_{0} \in U_{0}$ then dist $\left(z_{j}, \Lambda_{c}(h)\right) \rightarrow 0$ as $j \rightarrow \infty$.

Clearly, $\Lambda_{c}(h)$ is contained in the center-space $X$ for Example 2. In the general case we will cut $\Lambda(h)$ with $M_{h, c, \text { loc }}$ to obtain

$$
\Lambda_{c}(h)=M_{h, \epsilon, \text { boc }} \cap \Lambda(h) \text {. }
$$




\section{Theorem 7.3}

Under the assumptions (1.3), (1.4) let $z=0$ be asymptotically stable for (1.1). Let $\Lambda(h)$ be an attracting set for (7.6) with properties (P0) to (P3), the existence of such a set being guaranteed by [4]. If $\epsilon>0$ is fixed and $h \leq h_{0}(\epsilon)$ then $\Lambda_{c}(h)$ has properties (P1), (P2)' and (P3).

Proof:

1. Fix $\epsilon>0$ and choose $h_{0}$ so small that

$$
\Lambda(h) \subset B_{\epsilon / 4}(0)
$$

for $h \leq h_{0}$. Clearly $\Lambda_{c}(h)$ is compact. Let $z_{0} \in \Lambda_{c}(h)$, then $z_{1} \in \Lambda(h)$; in particular $z_{1} \in B_{\epsilon / 4}(0)$. Thus $z_{1} \in M_{h, \epsilon, \text { loc }}$ since this manifold is locally invariant. This shows that (P1) holds.

2. To show that $\Lambda_{c}(h)$ attracts nearby trajectories let

$$
z_{0}=\left(x_{0}, y_{0}\right) \in U_{0}
$$

denote a starting point. Since $\Lambda(h)$ absorbs $z_{j}$ there exists $J$ with

$$
z_{j} \in \Lambda(h) \subset B_{\epsilon / 4}(0) \text { for } j \geq J .
$$

We can apply Theorem 7.3 with starting point $z_{J}$ and find

$$
\left\|y_{j}-u_{h, \varepsilon}\left(x_{j}\right)\right\| \rightarrow 0 \text { as } j \rightarrow \infty
$$

Let $z_{j_{k}}$ denote a convergent subsequence of $z_{j}$ :

$$
z_{j_{k}}=\left(x_{j_{k}}, y_{j_{k}}\right) \rightarrow(\bar{x}, \bar{y})=\bar{z} \in \Lambda(h)
$$

By continuity, $u_{h, \epsilon}\left(x_{j_{h}}\right) \rightarrow U_{h, \epsilon}(\bar{x})=: \bar{u}$, and $(\bar{x}, \bar{u}) \in M_{h, \epsilon, \text { loc }}$. But then (7.7) shows $\bar{y}=\bar{u}$, i.e.

$$
\bar{z} \in M_{h, \epsilon, \text { loc }} \cap \Lambda(h)=\Lambda_{c}(h),
$$

and consequently

$$
\operatorname{dist}\left(z_{j_{k}}, \Lambda_{c}(h)\right) \leq\left\|z_{j_{h}}-\bar{z}\right\| \rightarrow 0
$$


The final result

$$
\operatorname{dist}\left(z_{j}, \Lambda_{c}(h)\right) \rightarrow 0 \text { as } j \rightarrow \infty
$$

follows by a standard compactness argument.

3. Since $0 \in \Lambda_{c}(h) \subset \Lambda(h)$ the property (P3) is obvious for $\Lambda_{c}(h)$.

Remark: The result of Theorem 7.3 is - for the special case of a singleton set $\Lambda=\{0\}-$ an improvement over the result in [4] since the set $\Delta(h)$ constructed in [4] always has a nonempty interior whereas the set $\Lambda_{c}(h)$ constructed in the present paper generally lies in a manifold of lower dimension: exponentially decaying parts are 'split off'. This raises a more general question. Suppose $\Lambda \subset \mathbb{R}^{n}$ is an attracting set for a system of ordinary differential equations and suppose we can apply the result in [4] to obtain an approximating attracting set $\Lambda(h)$ for a given one-step method. Now suppose $\Lambda$ lies in a submanifold $M$ of $\mathbb{R}^{n}$ which attracts nearby trajectories at an exponential rate. Under quite general assumptions - see [3] - the manifold $M$ will persist under small perturbations, thus it can be expected that the one-step method has an approximating invariant manifold $M_{h}$ of the same dimension as $M$. Will this allow us to conclude the existence of an attracting set $\Lambda_{c}(h)$ lying in $M_{h}$ which approximates $\Lambda$ ?

\section{ACKNOWLEDGMENTS}

This work was initiated while the first author was a Visiting Associate at Caltech. We would like to thank the Applied Mathematics Department, especially Prof. H.O. Kreiss, for providing this opportunity. We would also like to thank Prof. H.B. Keller for suggestions which led to improvements of the original manuscript. This research was supported by the Office of Naval Research under contract No. N00014-83-K-0422 and the U.S. Department of Energy under grant No. DE/AT/03/76ER/72012.

\section{REFERENCES}

1. W.-J. Beyn, On the numerical approximation of phase portraits near stationary points. SIAM J. Numer. Anal., to appear. 
2. F. Brezzi, S. Ushiki, H. Fujii, Real and ghost bifurcation dynamics in difference schemes for ODEs. In: Numerical Methods for Bifurcation Problems (T. Küpper, H.D. Mittelmann, H. Weber, eds.), pp. 79-104. Birkhāuser Verlag, 1984.

3. N. Fenichel, Persistence and smoothness of invariant manifolds for flows. Indiana University Math. J., Vol. 21, No. 3 (1971), pp. 193-226.

4. P.E. Kloeden, J. Lorenz, Stable attracting sets in dynamical systems and their one-step discretizations. SIAM J. Numer. Anal., Vol. 23, No. 5 (1986), pp. 986-995.

5. J. Sijbrand, Properties of center manifolds, AMS Transactions, Vol. 289, No. 2 (1985), pp. 431-469.

Received: August, 1986

Accepted: January, 1987 